\title{
A Comprehensive Study of DYMO Routing Protocol
}

\author{
Saloni Sharma \\ Scholar \\ RIMT, Mandigobindgarh, \\ Mtech (CSE)
}

\author{
Anuj Kumar Gupta \\ Head of the Department \\ RIMT, Mandigobindgarh \\ Mtech (CSE)
}

\begin{abstract}
Manet is one of the most popular and frequently emerging type of wireless network. Because of its increasing usability different types of routing protocols have been designed. These routing protocols are designed basically to meet the need of manets such as flexibility, mobility, security etc. Among all the different type of routing protocol DYMO routing protocol is new which is developed by IEFT which is also referred to as a successor of AODV routing protocol. DYMO is considered as the better routing protocol in mobile nodes network. In this paper we will discuss its emergence, its working and its characteristics which make it different from other routing protocols.
\end{abstract}

\section{Keywords}

MANETs, Security, Routing Protocol, AODV, DYMO.

\section{INTRODUCTION}

A mobile adhoc network is a collection of mobile nodes with no fixed infrastructure and with no permanent network. All nodes in the network are allowed to move freely throughout the network and have a permissive transmission range. Due to nodes limited transmission range, each node require other neighbor node to forward the packets. [3] Different challenges faced by manets:-

- Infrastructure less network - Due to its infrastructure less network it becomes difficult to detect the malicious nodes or faults.[9]

- $\quad$ Packet loss - Due to its mobile nature the possibility of losing packets while forwarding increases.

- Mobile nodes - Since nodes can easily join or leave the network so they tend to behave maliciously easily.[9]

- $\quad$ Security - Security is a major challenge faced by manets due to open exposure to nodes to attackers, lack of infrastructure, dynamic topology etc. [5]

Routing in manets is a critical issue, since each node acts as a router. To preserve the security of manets from different types of attacks, a routing protocol must fulfill certain requirements. [3] A routing protocol specifies how particular communication is carried between different routes. Thus, the routing algorithm helps in selecting the choices of routes that can be followed. Accordingly there are three major types of routing protocols - reactive (on - demand), proactive (table driven) and hybrid. In the paper the reactive routing protocols and DYMO routing protocol has been discussed in details.

\section{REACTIVE ROUTING PROTOCOL}

Routing in manets is divided into 3 categories as per diagram below:

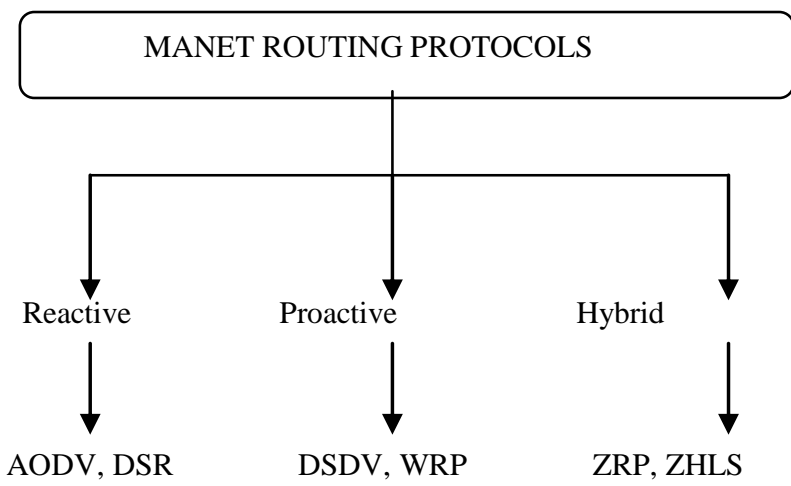

Fig1. Types of manet routing protocols

Reactive routing protocols are also referred as on - demand routing protocols. They obtain the necessary route when it is required by using a connection establishment process. [1] Like pro-active routing protocols they do not exchange routing information periodically. They do not maintain any routing information unless there is a connection. Whenever any node in a particular network wants to send packet or information to any other node in the network then reactive protocol searches for the appropriate route in the network in on - demand manner and then establishes the connection.

The reactive routing protocols are basically designed to overcome the short comings of the pro - active routing protocols Reactive protocols overcome the increased overhead problem of proactive routing protocols. In reactive routing protocols a route maintenance process is implemented to maintain a route until the destination is no longer available. Hybrid routing protocols are basically a combination of both reactive and pro-active routing protocols. AODV (adhoc on demand distance vector), DSR (distance source routing), 
DYMO (dynamic mobile on demand routing protocol are some of the reactive routing protocols.

\section{DYMO}

DYMO (dynamic manet on-demand routing protocol) is a newly proposed routing protocol by IETF Internet draft [9], it is in its twenty - fifth version [11] and is still in progress. [5] It is proposed by Perkins \& Chakeres. Its first internet draft was released in 2005. It is a successor of AODV routing protocol, therefore it shares many of it features and is also called AODVv2. [10] With each of its update it is improving and becoming better. Basically it does not add any extra features or does not extend the AODV routing protocol, the basic working operation is same. It just simplifies the process of routing. DYMO has somewhat simpler design as compared to AODV. Some of the characteristics of DYMO over AODV are -

- DYMO has a lower routing overhead than AODV.

- Using path accumulation function it simplifies the protocol implementation.

- The basic routing process of DYMO involves route discovery and route maintenance.

- DYMO is basically an improvement over AODV protocol as for AODV every node records its address to the route request while sending it to the destinations. [ 4]

- DYMO can be operated at other layers other than network layer. [8].

- DYMO protocol can be used in both IPv4 and IPv6 network and can also be operated with the internet.

- DYMO is a simple and better routing protocol for multihop networks.

\section{WORKING OF DYMO}

Similar to AODV, the basic operation of DYMO is also route discovery and route maintenance. Since DYMO can work as both reactive and proactive routing protocol, its features make it better for both IPv4 and IPv6 scenarios. In this protocol routing information of active sources and destination is maintained. This protocol is also suitable for scalability. [8]
Let us explain the basic operation between the DYMO and AODV routing protocol diagrammatically.

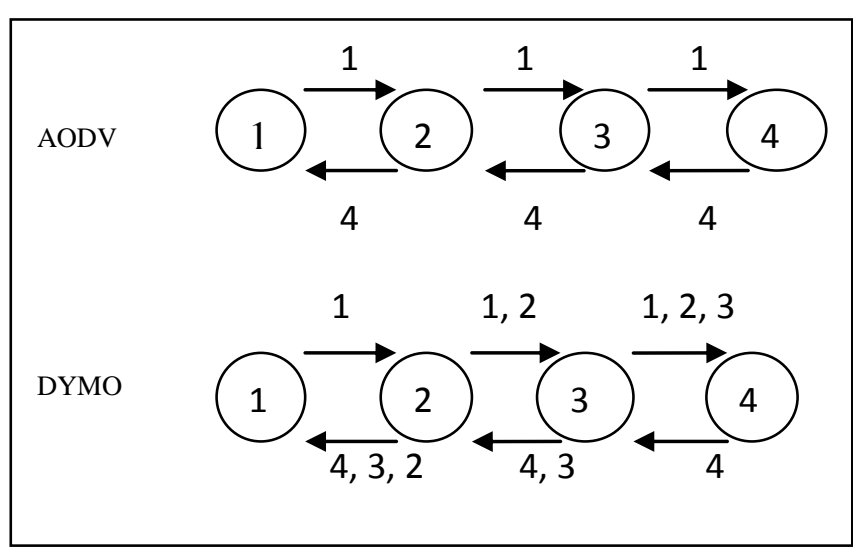

Fig2. A diagramatic description of difference between AODV and DYMO routing protocol when node 1 wants to communicate with node 4 .

As per the above diagram the DYMO routing protocol performs the path accumulation function. The entire process is similar to that of AODV except that while broadcasting the RREQ (which are signified with the help of arrows) the intermediate node will attach its address to the message. Every intermediate node that carries the RREQ message makes a note of the backward path. No such path accumulation happens in AODV. This path accumulation function helps DYMO in having a reduced routing overhead as compared to AODV.

\subsection{DYMO Routing Table}

According to the DYMO IETF draft 25, [11] route table entry consists of following fields:

Address: The length of the prefix.

Sequence Number: The sequence number associated with a route table entry.

Next Hop Address: An IP address of the adjacent DYMO router on the path towards the route address.

Last Used: The time any particular route was last used.

Expiration Time: The time at which the particular route expires.

Broken: A flag indicates if the particular route is broken. The flag is set to true if the next - hop becomes unreachable.

Metric Type: The type of metric for the route towards route address. 
Metric: The cost of route towards route address.

Thus, a route table entry (i.e. route) may be in one of the following states.

Active: An active route is currently in use for forwarding packets.

Idle: A route which is not in use currently is called idle route. Although that idle route can be used for forwarding packets on future

Expired: If a route is idle for a longer period of time it is considered expired. Thus, it may be no longer used for forwarding packets.

Broken: A route which is broken cannot be used for forwarding packets but still has valid destination sequence number information.

Timed: The expiration of a timed route is controlled by the route. Until that time a timed route can be used for forwarding packets, afterwards the route must be expired.

\subsection{Route Discovery}

DYMO route discovery is very much similar to that of AODV except the path accumulation function. The first step in the route discovery process is to initiate forwarding RREQ throughout the network to find a suitable route to the destination node. Thus to initiate the communication with RREQ i.e. route request messages the source forwards them to their neighbors. The RREQ (route request) and RREP (route reply) are the control messages or the routing messages which are used to forward the communication within the nodes in a network. The sequence number is automatically incremented before it is added to the RREQ. If the neighbor has any particular route to the destination it replies with the RREP message else it broadcasts the message. As per in DYMO the difference is that while in the route discovery process the intermediate node will attach its address to the message. Thus, every intermediate node that initiates the RREQ message makes a note to the backward path i.e. the backward address is recorded. [10]

We extend the illustration of the route discovery process with the help of the figure.

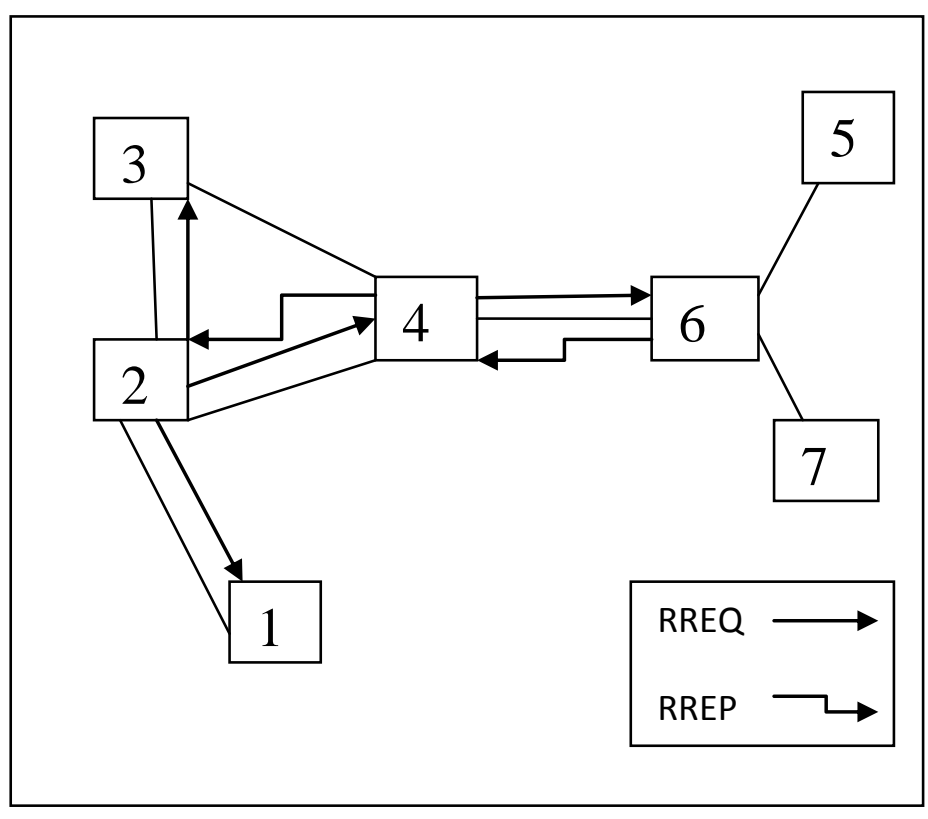

Fig3. DYMO route discovery process. Node 2 wants to communicate with node 6 . Each node forwarding the RREQ creates a reverse route to 2 used when sending back the RREP.

As shown in the figure above node 2 is the source node and node 6 is the destination node. Thus, node 2 sends the RREQ message to its neighbours i.e. node 1,3 and 4 . In the RREQ message node 2 includes its own address and sequence number. Finally, a hop count for the source node is added. The most important part is the address of the target. Each node forwarding an RREQ contains its own address, sequence number, prefix and gateway information to the RREQ. In the above diagram the source node 2 communicates to node 6 via node 4. Upon sending the RREQ the source node waits for the RREP message from the destination. If no RREP is received within the wait time, the source node may try again to discover the route by sending another RREQ after sometime. The RREP message is thus created, containing information about the destination node i.e. node 6 .

Summary of route discovery can be explained as below:

- $\quad$ Node 2 wants to communicate with node 6. Node 2 begins route discovery and forwards the RREQ. When node 4 receives the RREQ, it installs the route to node 2 . After node 4 forwards the RREQ it adds its own address to the RREQ.

- When the destination node i.e. node 6 receives the RREQ it contains all the three addresses the source, the destination and the intermediate node 4 . Thus node 6 creates an RREP as a response which is sent back with the reverse route. Similar to RREQ, the RREP also follows the path accumulation function and every node forwarding the RREP adds its own address to the message. 


\subsection{Route Maintenance}

Each and every node transmitting a packet is responsible for enquiring that the next neighbor receives the packet. Let us illustrate it with the help of diagram below.

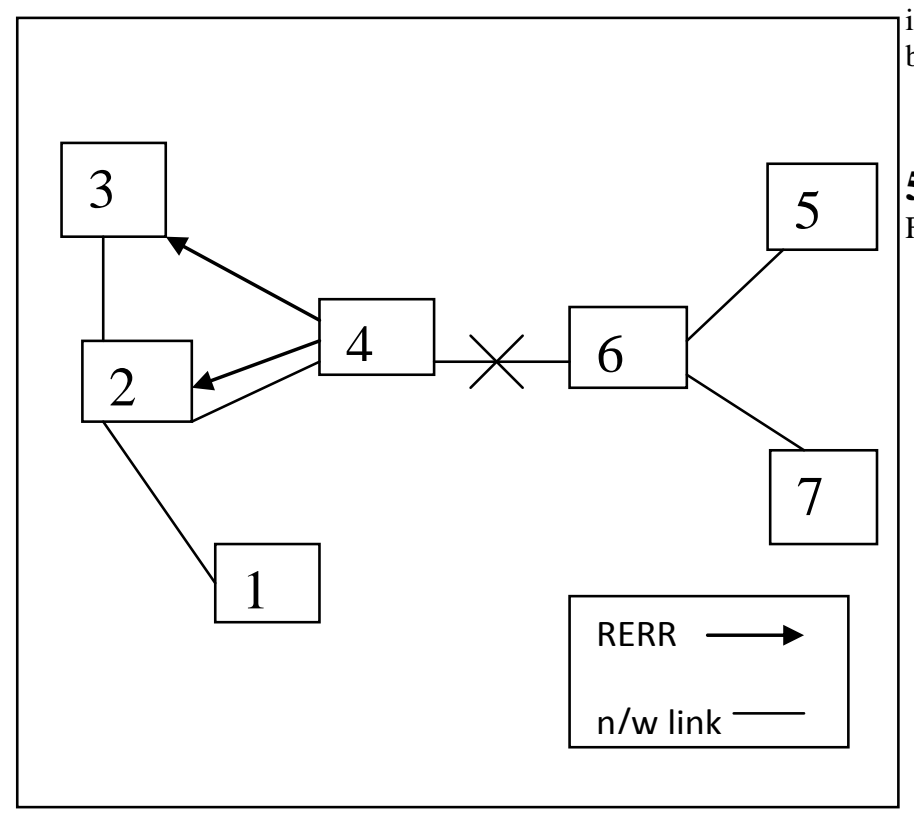

Fig4. Generation of RERR messages. The link between node 4 and node 6 breaks and node 4 sends RERR message to other nodes.

Route maintenance consists of two steps - first, it detects that a node to the route is no longer exists. Second, it will remove that particular route and will send the link failure i.e. RERR message to all the neighbors that are actively using the route. The RERR message contains the IP address of each destination which has become unreachable due to the link break [10]. The affected source node then either stop sending data or reinitiate the route discovery process by sending a new RREQ message. In above figure the node 6 is broken or has moved outside the transmission range of node 4 . Node 4 then returns RERR message to all the neighbors that use the route to node 6 .

\subsection{Optional Features}

According to the draft(25) [11] some additional features of DYMO over AODV are below. These features are usefull in the network with greater mobiliy:

$>$ Expanding rings multicast

$>$ Intermediate RREP

$>$ RREP_ACK

$>$ Message aggregation

$>$ Reporting multicast unreachable nodes

\subsection{Characteristics of DYMO}

Few characteristics of DYMO are:

1. DYMO improves the performance of the network by mutipath routing.

2. It simplifies the process of AODV with source routing and path accumulation technique.

3. As a reactive routing protocol DYMO does not store the network topology. [6]
4. DYMO can easily adapt to wide range of traffic patterns in a network.

5. Since DYMO maintains a very little routing information it is therefore considered as quite a memory efficient protocol because a much lesser amount of memory is been used.

\section{ADDITIONAL FEATURES OF DYMO}

Few additional features of DYMO are discussed below:

* Adjacency Monitoring - DYMO does not use any kind of HELLOW messages to make sure that the adjacent neighbors are active or not. When two nodes try to communicate with each other the link layer of the third node becomes active and checks whether it can communicate or not. [10]

* Path Accumulation - During the route discovery process, the originator i.e. the source initiates the RREQ message throughout the network to find a valid route to the destination. Upon receiving the RREQ the intermediate node containing the route to the destination records the route to the originator and thus rebroadcasts the RREQ. Adding its own address to the destination. This process is called as path accumulation function. Similar process takes place with the backward route. While the destination prepares the RREP, each intermediate node adds its address to the route. The path accumulation function allow nodes to have the proper knowledge of the routing. This helps routes to know about other valid routes without initiating any route requests. [10] This path accumulation function helps in reducing the routing overhead of the network.

* Multipath extension to DYMO - Multipath extension is used so that even if one path fails or there is some link error the data can be routed through another path. In on demand routing protocols when the source has to send some packets to any particular destination in a network, it sends the RREQ to the network. The destination then replies by sending an RREP. If any link breaks in the route the RERR message is sent back to the source. Followed by the route request process takes place again. In multipath concept the node establishes more than one path with different ways and different standards. Based on DYMO multipath protocols are designed which ensures the usage of multiple paths towards the destination.

* Secure DYMO - SEDYMO is a mechanism to secure a dynamic multihop adhoc routing protocol. Digital signatures and hash functions are considered to ensure the security of the protocol. This protocol extension ensures integrity and authentication of the network. SEDYMO deals with public key cryptography. 


\section{COMPARISON TABLE OF DIFFERENT REACTIVE PROTOCOLS}

The formal comparison of three different reactive routing protocols are given below:

Table 1. Comparison of DSR, AODV and DYMO protocols

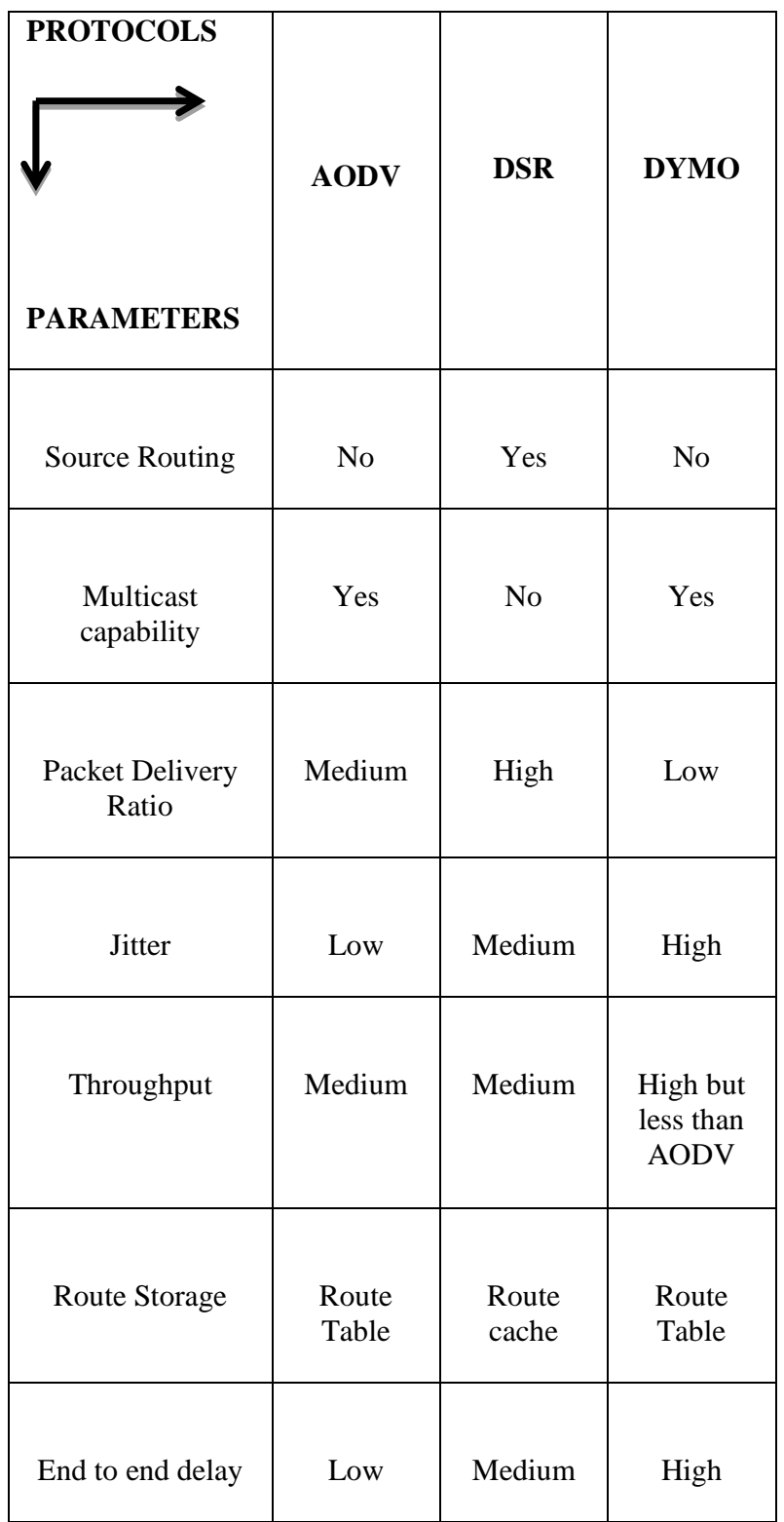

As per the table the comparison of different routing protocols has been done. The three frequently used on - demand routing protocols are taken into consideration i.e. AODV, DSR and DYMO. DSR is also a reactive routing protocol which follows source routing. Among AODV, DSR and DYMO it can be seen that DYMO is better and technically advanced routing protocol due to obvious reasons, its path accumulation function. Due to DYMO's multipath capability and path accumulation function the throughput is much lesser than AODV and DSR. Basically the choice of protocols in mobile adhoc environments also depends on the type of the application used. Even the packet delivery ratio is lowest in case of DYMO. For a network which requires end to end delay AODV is suitable. Although the parameters fluctuate as the number of nodes changes. Thus, the behavior of each protocol must be discussed in detail in different environments. More of DYMO routing protocol should be discussed in presence and absence of different types of attacks so that its applicability and suitability is much more clearer.

\section{CONCLUSION}

In this paper different features of mobile adhoc networks, their respective routing protocols and mainly the newly existed DYMO routing protocol are discussed. Since DYMO includes some of the best features of routing among other routing protocols, its additional features are discussed in detail. A comparison between different reactive rouitng protocols has been done in order to evaluate which routing protocol works better. A lot of research and development work on DYMO is still required.

\section{ACKNOWLEDGMENTS}

The authors sincerely thank to their institution for giving this opportunity to work and providing environment to study and for research. The authors wish to thank the reviewers and editors for their valuable suggestions and expert comments that help improve the contents of paper.

\section{REFERENCES}

[1] Loay Abusalah, Ashfaq Khokhar, and Mohsen Guizani "A Survey of Secure Mobile Ad Hoc Routing Protocols" in proceedings of ieee communications surveys \& tutorials, vol. 10, no. 4, fourth quarter 2008.

[2] Davide Cerri and Alessandro Ghioni "Securing AODV: The A-SAODV Secure Routing Prototype" in proceedings of IEEE Communications 01636804/08/\$25.00 @ 2008 IEEE.

[3] Anuj K. Gupta "Secure Routing Techniques for mobile adhoc networks" in proceedings of 2009 IEEE International Advance Computing Conference (IACC 2009) Patiala, India, 6-7March 2009.

[4] A.Boomarani Malany and V.R.Sarma Dhulipala and RM.Chandrasekaran "Throughput and Delay Comparison of MANET Routing Protocols" as in proceedings of ICSRS September,2009.

[5] Sudhir Agrawal, Sanjeev Jain, Sanjeev Sharma and Roopam Gupta "Mobility based Performance Analysis of AODV and DYMO under Varying Degree of Node Misbehavior" ai in proceedings of IJCA,September 2011.

[6] Archie Budhiraja and Roopali Garg "Performance Comparison of Dynamic Mobile ad-hoc network ondemand multipath routing protocol with AODV" as in proceedings of International Journal of Scientific \& Engineering Research,November-2011

[7] Anuj K. Gupta, Harsh Sadawarti, and Anil K. Verma "Review of Various Routing Protocols for MANETs" in proceedings of International Journal of Information and Electronics Engineering, Vol. 1, No. 3, November 2011.

[8] Richa Agrawal, Rajeev Tripathi and Sudarshan Tiwari “ Performance Comparison of AODV and DYMO MANET Protocols under Wormhole Attack Environment 
" as in proceedings of International Journal of Computer Applications April 2012 .

[9] Saloni Sharma and Anuj Kumar Gupta "A Comprehensive Review of Security Issues in Manets" as in proceedings of IJCA,2013

[10] Jatinder Pal Singh and Anuj Kr. Gupta "A Review on Dynamic MANET On Demand Routing Protocol in MANETs" as in proceedings of International Journal of Advanced Trends in Computer Science and Engineering, march 2013.

[11] C. Perkins and I. Chakeres Mobile Ad hoc Networks Working Group Internet-Draft Futurewei Intended status: Standards Track I. Chakeres Expires: July 8, 2013 Dynamic MANET On-demand (AODVv2) Routing draft-ietf-manet-dymo-25.

[12] Yogesh Chaba, R. B. Patel, and Rajesh Gargi "Efficient Multipath DYMO Routing Protocol with Gateway Selection for Hybrid MANETs" as in proceedings of International Journal of Computer Theory and Engineering.

[13] Bharat Bhushan, Shailender Gupta and C.K.Nagpal “ Comparison of on Demand Routing Protocols" as in proceedings of I.J. Information Technology and Computer Science, 2013.

[14] Sukant Kishoro Bisoyi and Sarita Sahu "Performance analysis of Dynamic MANET On demand (DYMO) Routing protocol" as in proceedings of IJCCT.

[15] Richa Vats and Dr. Yusuf Mulge "Star, Dymo and Zrp routing protocols of manet" as in proceedings of int.j.computer technology \& applications, may-june 2013.

[16] Anuj K. Gupta, Harsh Sadawarti and Anil K. Verma "Implementation of dymo routing protocol" as in proceedings of International Journal of Information Technology, Modeling and Computing (IJITMC), May 2013.

[17] Mayuri D. Dondal and Prof. V.S.Jadhav "Manet performance evaluation based on Aodv, Olsr and Dymo routing protocol" as in proceedings of International Journal of Emerging Trends in Electrical and Electronics (IJETEE)July-2013.

[18] Christoph Sommer · Isabel Dietrich · Falko Dressler "Simulation of Ad Hoc Routing Protocols using OMNeT++ A Case Study for the DYMO Protocol" as in proceedings of $($ ) Springer. 